\title{
Evaluation of Kindlin-1 and Ki-67 immunohistochemical expression in primary cutaneous malignant melanoma: a clinical series
}

\author{
Luciana El Halal Schuch ${ }^{1,2}$, Munique Mendonça Azevedo ${ }^{1}$, Roque Furian², Péttala Rigon ${ }^{1,2}$, Keli Cristine Reiter ${ }^{1}$, \\ Isabel Crivelatti ${ }^{3}$, Felice Riccardi ${ }^{2}$ and Claudia Giuliano Bica ${ }^{1 *}$ (D)
}

\begin{abstract}
Background: The capacity for prognostic prediction of cutaneous melanoma, one of the most aggressive cancers, is still difficult due to the tumor heterogeneity and lack of reliable tumor markers. The objective of this study is to correlate, through immunohistochemistry, a Ki-67 and Kindlin-1 staining in malignant melanomas with the prognosis of the disease.

Methods: A historical cohort study. Immunohistochemistry, using mouse anti-human Kindlin-1 and Ki-67 monoclonal antibodies, was performed using tissue blocks from primary cutaneous melanoma patients treated between 2006 and 2014 at our institution. Information regarding pathological data and outcomes were retrieved from medical records. Statistical analyses were conducted in SPSS version 18.0.

Results: Thirty patients were included. The median age was from $50.93 \pm 15.31$ years old. The expression of Ki-67 was detected in all patients with primary cutaneous melanoma, while Kindlin-1 was negative in two. Kindlin expression was not significantly correlated with Ki-67 expression by Spearman's rank correlation analysis $(P=0.46)$, as well as the expression of both markers and the clinical stage $(P=0.34$ and 0.18 , respectively). Breslow, Clark and mitotic rate were significantly correlated with AJCC stage $(P=0.001)$.
\end{abstract}

Conclusion: Other studies investigating clinical evolution are needed to further test the potential of these markers as possible prognostic markers.

Keywords: Melanoma, Kindlin, Ki-67; biomarkers, Immunohistochemistry

\section{Introduction}

Cutaneous malignant melanoma is a tumor originating from skin melanocytes, and its incidence in individuals of European origin continues to rise worldwide. In 2016, approximately 76,380 new cases were diagnosed in the United States [1]. In addition, there are approximately 3.03 and 2.59 new cases per 100,000 people for men and women, respectively, in Brazil. The three southernmost states of Brazil have the highest proportion of individuals of Northern European Caucasian ancestry, with

\footnotetext{
* Correspondence: claudia@ufcspa.edu.br

'Universidade Federal de Ciências da Saúde de Porto Alegre, Porto Alegre, Brazil

Full list of author information is available at the end of the article
}

approximately 6.96 new cases per 100,000 men and 6.5 per 100,000 women in 2016 [2,3]. Melanomas are one of the most aggressive malignant neoplasms. Moreover, the clinical course is often unpredictable, and despite improved options due to targeted therapies, metastatic melanoma is difficult to treat [4-6].

The staging system proposed by the American Joint Committee on Cancer (AJCC) is currently used for melanoma classification, prognostic prediction and patient management [7]. Tumor invasion (Breslow thickness) has become the gold standard for stratifying patients according to the risk of metastatic disease. However, it has been noted that in some cases, tumor thickness is not an accurate indicator of biologic behavior [8]. For example, 
early-stage melanomas are clinically heterogeneous, with a subset exhibiting high-risk behaviors; approximately $5 \%$ of stage I melanomas metastasize early and eventually cause death [9]. Surgical excision of localized tumors (AJCC stages I and II) may definitively treat many patients. For these patients, the overall 5-year survival rate is approximately $80 \%$, suggesting that nearly $20 \%$ of patients may have micrometastatic disease at the time of diagnosis [10].

Thus, identifying biomarkers for use in conjunction with traditional cancer staging and prognosis might improve early diagnosis and patient care. Despite efforts to date, reliable biomarkers are still lacking [11]. Nonetheless, a prognostic association has been shown between the mitotic index and thin melanoma [12], and the Ki-67 index has been studied as a prognostic marker. However, the prognostic value of proliferative activity in cutaneous malignant melanoma remains unclear. Indeed, there are divergent findings among studies about the use of the Ki-67 index or conventional prognostic factors (sex, location, level of invasion, tumor thickness, ulceration, mitotic count, prognostic index and clinical stage), and some studies indicate that the Ki-67 index does not offer additional prognostic information for localized cutaneous malignant melanoma [13].

Immunohistochemical biomarkers able to provide additional information about disease progression have been extensively evaluated [14]. Unfortunately, none have yet been incorporated into clinical practice. Some of these biomarkers have demonstrated statistical significance as prognostic markers in the research setting, though there has been no systematic analysis of molecular biomarkers in an attempt to identify those capable of refining subgroups for individual AJCC stages $[15,16]$.

\section{Kindlin-1}

Kindlins have been investigated as cancer biomarkers for more than a decade. Fermitin-family-member-1 (FERMT1, Kindlin-1) is an epithelial-specific regulator of integrin functions that is associated with Kindler syndrome, a genetic disorder characterized by skin blistering, atrophy, and photosensitivity. The possible role of Kindlin-1 in cancer remains unknown [17].

The most studied function of kindlins is their role in integrin activation. The term integrin was coined more than three decades ago to designate a family of cellsurface adhesion receptors, and the interrelationship between integrins and cancer pathology inevitably led to consideration of the role of kindlins in cancer. Within the last 5 years, more than 70 publications have linked kindlins, integrins and cancer, including descriptions of an association between kindlin-1, skin cancer, and downregulation of Kindlin-3 expression in melanoma cells, which promotes metastasis [18, 19]. Furthermore,
Kindlin-1-deficient cells display a defect in B1 integrin activation and reduced cell proliferation, adhesion, polarity and motility. Most kindlin-related studies have focused on the relevance of kindlin to skin disease. Regardless, kindlins are also reported to have associations with a poor prognosis in both breast and lung adenocarcinoma, and studies have documented aberrant Kindlin-1 expression levels in cancers of epithelial origin. Patients who lack Kindlin-1 (Kindler Syndrome patients) may have an increased risk of squamous cell carcinoma [20].

Kindlin expression levels are reportedly elevated in $60 \%$ of lung cancers and $70 \%$ of breast cancers. Kindlins have also been associated with the pathology of glioma and pancreatic cancer [21, 22]. Although research has demonstrated a role for the Kindlin-1 antibody in the prognosis of other cancers, there are, to the best of our knowledge, no studies of this marker in primary melanoma.

\section{Ki-67}

The Ki-67 antigen was discovered in a study of specific nuclear antigens for monoclonal antibodies in Hodgkin cancer cells; the Ki-67 antigen is an important tool for quickly and reliably determining the proliferation rate of malignant tumor cells [5]. Ki-67 has been assessed with regard to diagnostic differentiation between a nevus and melanoma, and the Ki-67 proliferation index is higher for malignant melanocytic tumors than nevi and is higher in metastases than in primary melanomas. Despite these results, a low Ki-67 index for a malignant lesion does not eliminate the possibility of melanoma, as some malignant tumors proliferate at low rates, further demonstrating that more studies are needed [23, 24].

A previous study sought to investigate whether the Ki67 index is able to prevent false-negative diagnoses in melanomas. The authors proposed that a high cellular proliferation rate may be suggestive of melanoma and that the Ki-67 proliferation index might help prevent misdiagnoses of melanomas initially classified as benign or dysplastic nevi [25]. By comparing the prognostic impact of phosphohistone H3/MART1, Ki67/MART1, and hematoxylin and eosin staining, another study found alternatives to conventional detection of mitosis based on hematoxylin and eosin staining in the first two stages of primary cutaneous melanoma [26].

The aim of this study was to correlate Ki-67 and Kindlin-1 immunohistochemical staining in primary malignant melanomas with prognostic factors.

\section{Materials and methods}

\section{Patients and tissue samples}

We retrieved data for thirty patients from the files of patients diagnosed and treated at our institution (Irmandade Santa Casa de Misericórdia de Porto Alegre 

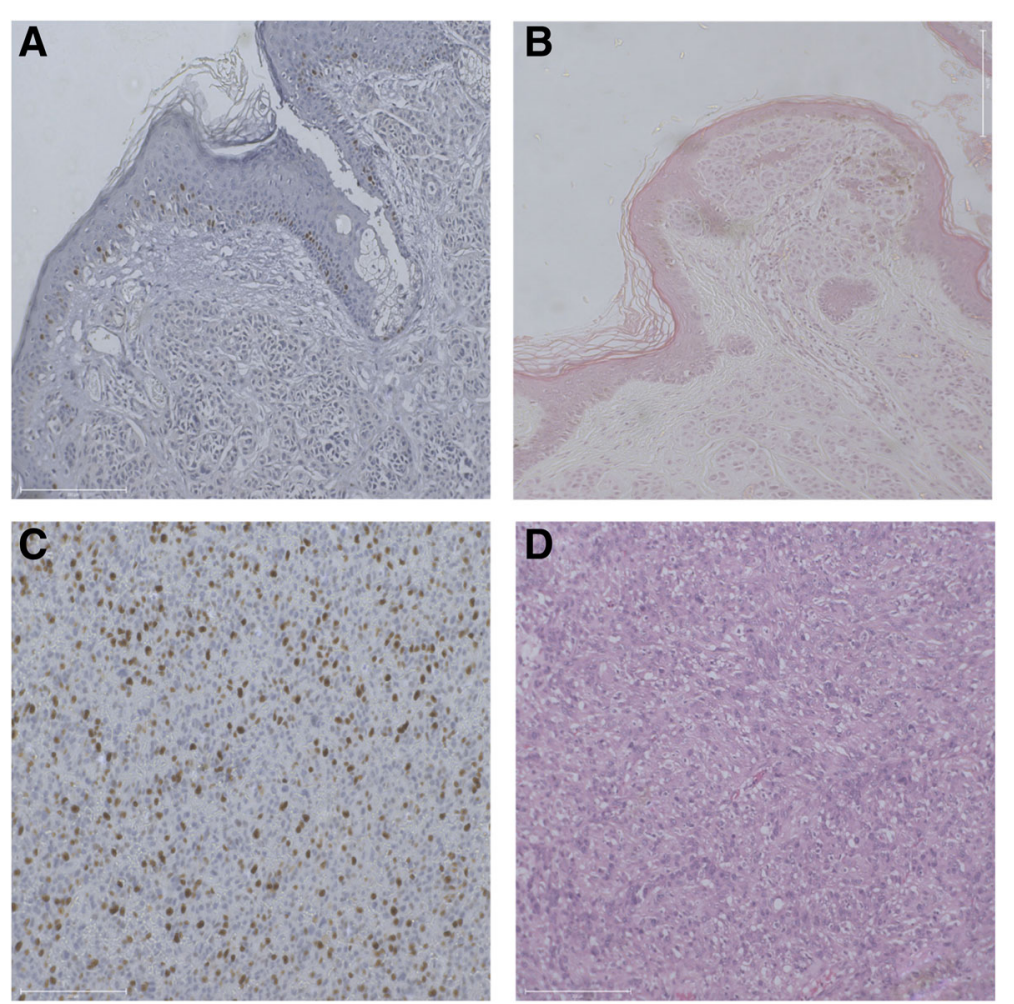

Fig. $1 \mathrm{H} \&$ E staining and Ki-67 expression by immunohistochemistry in primary cutaneous malignant melanoma (PCMM). The marker (brown) is expressed in the nuclei of PCMM cells. a: less than $1 \%$ of positivity and (b): corresponding to H\&E staining; $\mathbf{c}$ greater than $60 \%$ of positivity and (d) corresponding to H\&E staining

Hospital - ISCMPA, and Universidade Federal de Ciências da Saúde de Porto Alegre - UFCSPA) between July 2006 and September 2014; these patients all completed at least 2 years of follow-up. Clinical records were reviewed, and follow-up data were obtained from these and tumor registry records. A questionnaire about the main risk factors for the development of this neoplasia was created, and the following were recorded for the patients: sunburn episodes, familial and personal melanoma history, phenotypic characteristics and skin phototype.

Written informed consent was obtained from each patient according to the recommendations of the local ethics committee. This study was approved by the ethics committee of the hospital and the university where the study was developed (ISCMPA and UFCSPA, respectively).

For each patient, formalin-fixed paraffin-embedded tumor tissue (which was identified based on hematoxylin and eosin-stained sections and, when necessary, on S100 protein, melan A and HMB-45 immunohistochemistry sections) was obtained up from the pathology laboratory of ISCMPA. An independent histopathological review was performed by two pathologists on separate occasions.

\section{Immunohistochemistry (IHC)}

The most representative tumor area on the hematoxylin/eosin-stained slides was selected. Tissue sections $4-\mu \mathrm{m}$ thick were placed on salinized slides and dried at $60^{\circ} \mathrm{C}$ for $30 \mathrm{~min}$. The slides were then deparaffinized by three washes in xylene ( 5 min each) and rehydrated by successive washes in ethanol (absolute, 95, 70 and $50 \%$ ) and distilled water, according to our laboratory protocol. Antigen retrieval was performed in $\mathrm{pH} 9.0$ TRIS-EDTA buffer for $40 \mathrm{~min}$ at $98^{\circ} \mathrm{C}$. After heating and cooling for $20 \mathrm{~min}$, endogenous peroxidase activity was blocked by immersing the slides in 5\% hydrogen peroxide in methanol $(3 \times 10 \mathrm{~min})$. Finally, the slides were washed twice with $1 \mathrm{X}$ phosphate-buffered saline (PBS, pH 7.4) and incubated in 1\% BSA (bovine serum albumin) solution to block non-specific protein binding.

Primary antibodies against Ki-67 (1:50, mouse antihuman monoclonal Mib-1 - Dako ${ }^{\mathrm{R}}$, California, USA) and Kindlin-1 (1:100, mouse anti-human monoclonal antibody - Millipore ${ }^{\mathrm{R}}$, Massachusetts, USA) were added and incubated for $1 \mathrm{~h}$ (room temperature), after which the slides were placed at $4{ }^{\circ} \mathrm{C}$ overnight. Colon and breast tissue were used as positive controls. The negative controls were the same tissues without primary antibody incubation. After washing with PBS, the slides were 

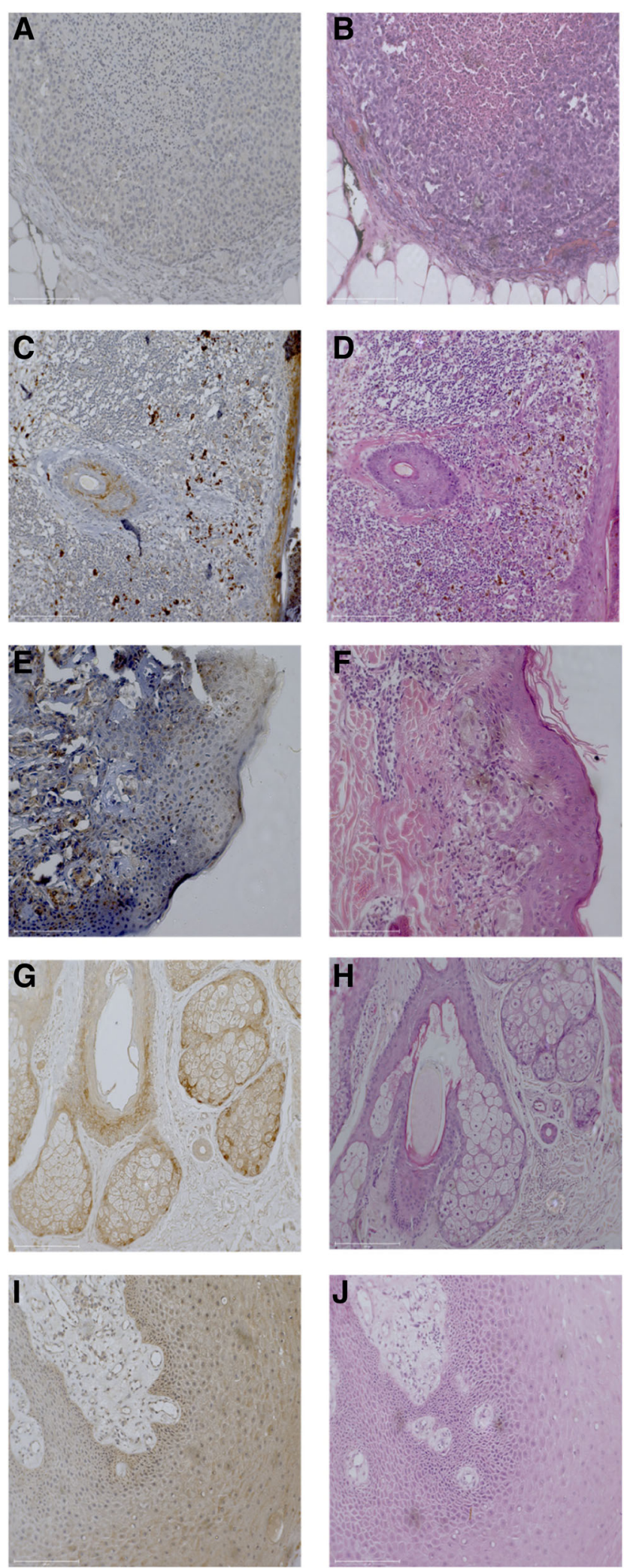

Fig. 2 (See legend on next page.) 
(See figure on previous page.)

Fig. 2 H\&E staining and kindlin-1 expression by immunohistochemistry in primary cutaneous malignant melanoma (PCMM). The marker (brown) is expressed in the citoplasm of PCMM cells. a negative and (b) corresponding to H\&E staining; $\mathbf{c}$ focal and (d) corresponding to H\&E staining; e multifocal and (f) corresponding to H\&E staining; $\mathbf{g}$ diffuse and (h) corresponding to H\&E staining; i 100\% positivity and (j) corresponding to H\&E staining

incubated with peroxidase-labeled polymer Picture $^{\mathrm{Tt}}$ Max HRP polymer conjugate broad spectrum (Life Technologies $^{\mathrm{R}}$, California, USA) and then with the DAB substrate chromogen (Dako ${ }^{\mathrm{R}}$, California, USA).

\section{Technical analysis}

The samples were evaluated by two researchers who determined the presence or absence of immunostaining. Staining was considered absent if no reaction was observed on the IHC sections. The quality (intensity, cell count and pattern) of Kindlin-1 and Ki-67 staining was compared using consecutive control sections, and the staining characteristics were recorded in a blinded method by two independent pathologists.

Digital images of the IHC results in three hotspots (points of maximum concentration of labeled cells) were captured [27]. For Ki-67, the pathologists performed a manual count of nuclear positivity in 500 cells at $400 x$ magnification for each hotspot [28]. The percentage of cells expressing Ki-67 was scored as a continuous variable, from 0 to $100 \%$. Kindlin-1 cytoplasmic qualitative staining intensity was scored as negative, focal, multifocal or diffuse. The scores of the two pathologists were compared, and any discrepancy was re-examined to achieve a consensus score. Figures 1 and 2 show some immunohistochemistry photomicrographs and their scores with each respective hematoxylin and eosinstained section (images captured by Invitrogen EVOS FL Auto 2.0 Imaging System).

\section{Statistical analysis}

SPSS version 23.0 software for Windows (SPSS Inc., IL, USA) was used for the statistical analysis. We used Mann-Whitney tests to determine associations between Kindlin-1 and Ki-67 expression and the clinicalpathological parameters of melanoma patients, including risk factors, AJCC stage, tumor thickness, ulceration, histologic subtype and tumor location. Correlation between Kindlin-1 and Ki-67 expression was analyzed using Spearman's rank correlation. $P<0.05$ was considered statistically significant.

\section{Results}

The patients included in the study were Caucasian, and the median age was $50.93 \pm 15.31$ years. Among the patients, $46.7 \%(14 / 30)$ were men and 53.3\% (16/30) women. The characteristics of the primary tumors are shown in Table 1.
Ki-67 expression was detected in all patients (100\%) with primary cutaneous melanoma, whereas Kindlin-1 expression was negative in 2 patients. Correlations between Kindlin-1 and Ki-67 expression and AJCC stages were assessed for all patients. The immunohistochemistry results are illustrated in Figs. 3 and 4.

The correlations between Kindlin-1 and Ki-67 expression and the clinicopathological features of the patients with primary melanoma are summarized in Table 2. Of the clinical risk factors, Kindlin-1 and Ki-67 expression did not correlate with skin phototype, eye color or childhood sunburn episodes $(P=0.51$ and $0.93, P=0.49$ and 0.68 , and $P=0.83$ and 0.07 , respectively). Among

Table 1 Clinicopathological features of primary melanoma tumor

\begin{tabular}{|c|c|c|}
\hline Variable & $N$ & $\%$ \\
\hline \multicolumn{3}{|l|}{ Tumor Site } \\
\hline Head/neck & 2 & 6.7 \\
\hline Trunk & 13 & 43.3 \\
\hline Upper limb & 9 & 30 \\
\hline Lower limb & 6 & 20 \\
\hline \multicolumn{3}{|l|}{ Histological subtype } \\
\hline Superficial spreading & 23 & 76.7 \\
\hline Lentigo maligna & 1 & 3.3 \\
\hline Nodular & 6 & 20 \\
\hline \multicolumn{3}{|l|}{ Clark's level } \\
\hline I & 5 & 16.7 \\
\hline$\|$ & 5 & 16.7 \\
\hline III & 5 & 16.7 \\
\hline IV & 15 & 50 \\
\hline \multicolumn{3}{|l|}{ Breslow tickness } \\
\hline Tis & 5 & 16.7 \\
\hline$\leq 1 \mathrm{~mm}$ & 12 & 40 \\
\hline $1.01-2 \mathrm{~mm}$ & 7 & 23.3 \\
\hline $2.01-4 \mathrm{~mm}$ & 4 & 13.3 \\
\hline$>4 \mathrm{~mm}$ & 2 & 6.7 \\
\hline \multicolumn{3}{|l|}{ Presence of ulceration } \\
\hline Yes & 5 & 16.7 \\
\hline No & 25 & 83.3 \\
\hline \multicolumn{3}{|l|}{ Mitosis ratio } \\
\hline Mean \pm SD & $2.23 \pm 2.97$ & \\
\hline Total & 30 & \\
\hline
\end{tabular}

Tis Tumor in situ, SD Standard deviation 


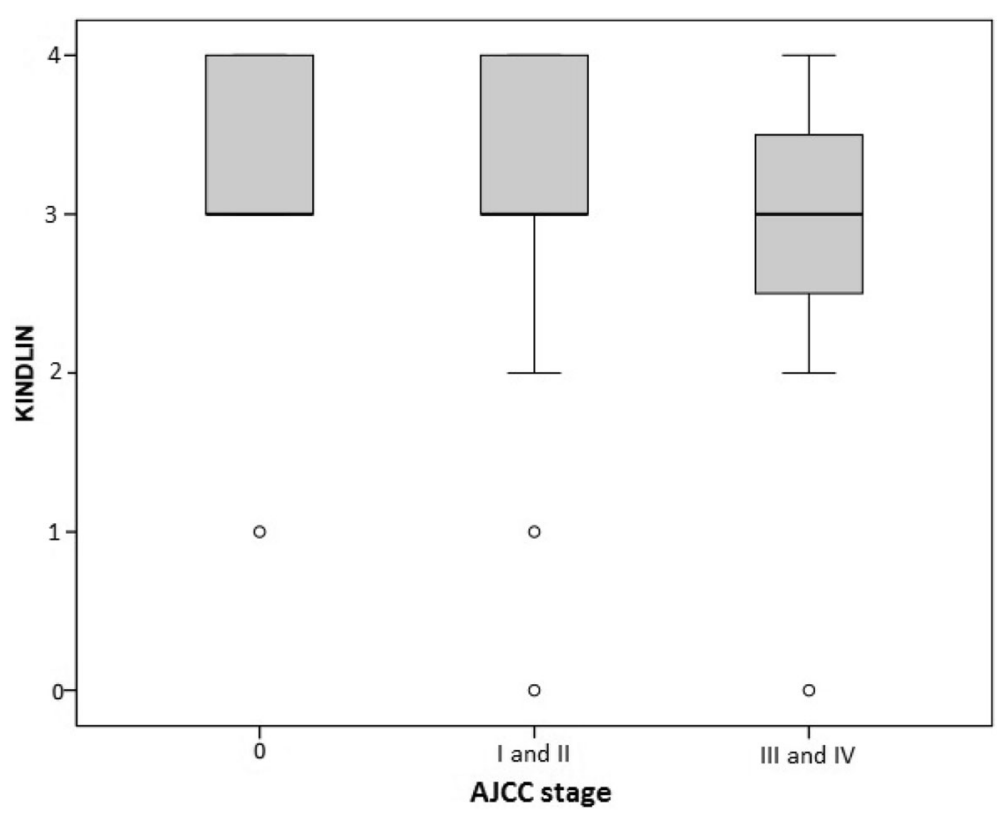

Fig. 3 Immunohistochemistry results for Kindlin-1 (intensity) and AJCC Stage (Kruskall Wallis Test; $P=0.89$ )

pathological factors, marker expression was not significantly associated with ulceration, sentinel lymph node status, Breslow thickness or mitotic rate $(P=0.66$ and $0.51, P=0.31$ and $0.37, P=0.57$ and 0.37 , and $P=0.37$ and 0.72 , respectively).

Moreover, according to Spearman's rank correlation analysis, Kindlin-1 expression did not correlate with Ki67 expression (Table 3, $P=0.46$ ), and there was also no correlation between the expression of both markers and clinical stage ( $P=0.34$ and 0.18 , respectively).

\section{Discussion}

The primary objective of this study was to evaluate Kindlin-1 and Ki-67 immunostaining in a series of patients with primary melanoma. Malignant melanoma is an aggressive skin cancer due to its rapid progression, propensity to

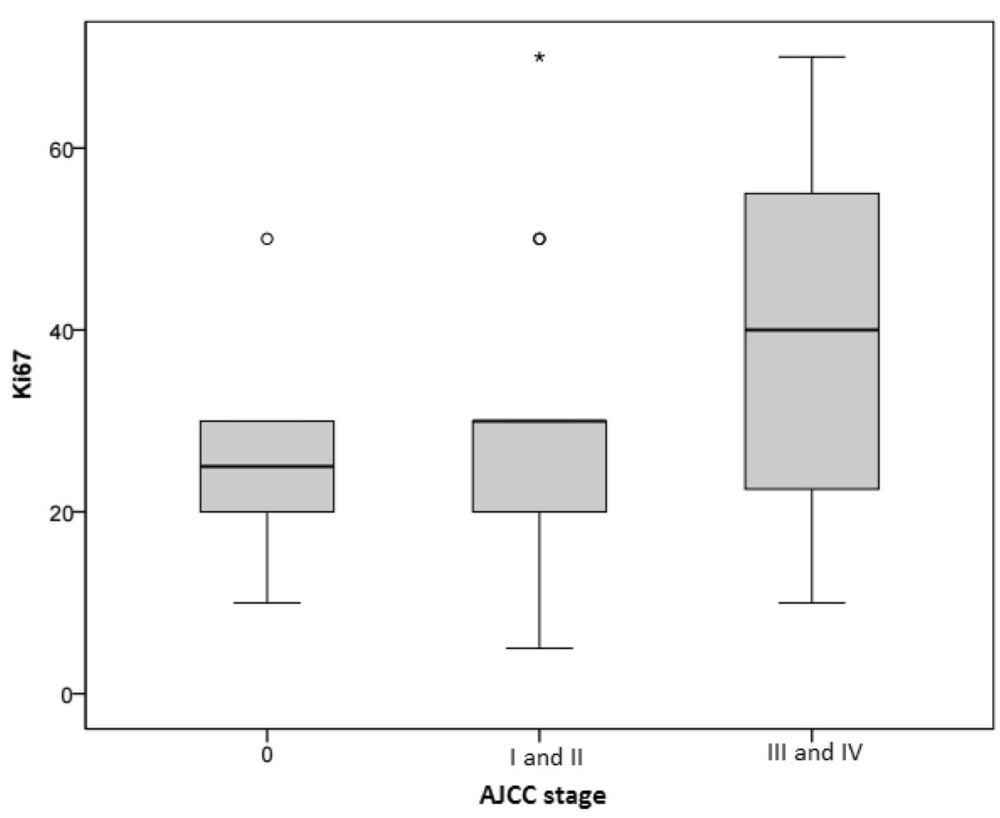

Fig. 4 Immunohistochemistry results for Ki-67 (\%) and AJCC Stage (Kruskall Wallis Test; $P=0.41$ ) 
Table 2 Association between biomarkers level and risk and prognostic factors for melanoma (Mann-Whitney test)

\begin{tabular}{|c|c|c|c|}
\hline \multirow[t]{2}{*}{ Variable } & \multirow[t]{2}{*}{$\mathrm{N}$} & \multicolumn{2}{|l|}{$P$} \\
\hline & & KINDLIN-1 & $\mathrm{Kl}-67$ \\
\hline Sunburn episodes & & 0.83 & 0.07 \\
\hline Yes & 17 & & \\
\hline No & 13 & & \\
\hline Skin phototype I/II & & 0.51 & 0.93 \\
\hline Yes & 15 & & \\
\hline No & 15 & & \\
\hline Eye color & & 0.49 & 0.68 \\
\hline Light & 13 & & \\
\hline Dark & 17 & & \\
\hline Presence of ulceration & & 0.66 & 0.51 \\
\hline Yes & 5 & & \\
\hline No & 25 & & \\
\hline SLN status & & 0.31 & 0.37 \\
\hline Positive & 5 & & \\
\hline Negative & 22 & & \\
\hline Breslow thickness & & 0.577 & 0.377 \\
\hline Mean \pm SD & $1.47 \pm 1.85$ & & \\
\hline Mitosis rate Mean \pm SD & $2.23 \pm 2.97$ & 0.379 & 0.727 \\
\hline Total & 30 & & \\
\hline
\end{tabular}

SLN Sentinel limph node, SD Standard deviation

metastasize, poor prognosis and intrinsic resistance to treatment. Because the disease is heterogeneous, tumor markers are needed to stratify these patients and to improve the accuracy of estimates of disease progression and survival. However, there is little information to date about markers for primary melanoma because there have been few studies correlating these markers with the clinicopathologic characteristics of this disease. Previous studies on tumor markers have compared dysplastic nevi, primary melanomas and melanoma metastases, finding correlations between differences in marker expression with disease progression.

Studies of tumor biomarkers are important because they facilitate disease stratification and benefit our understanding of tumor progression. Nonetheless, the most

Table 3 Correlation between stages and markers (Spearman's rank)

\begin{tabular}{ll}
\hline & Stages $^{\mathrm{a}}(N=30)$ \\
\hline Kindlin-1 Correlation coefficient & 0.178 \\
Sig. (2-tailed) & 0.347 \\
N Ki67 Correlation coefficient & 300.247 \\
Sig. (2-tailed) & 0.188 \\
N & 30 \\
\hline
\end{tabular}

${ }^{a}$ All stages $(0, \mathrm{I}, \mathrm{II}, \mathrm{III}$ and IV) valuable biomarkers are those that indicate response to treatment [14].

A previous study showed that Kindlin-1 expression in breast tumors is associated with lung metastasis [15]. Furthermore, some evidence suggests that Kindlin-1 is a potentially important clinical mediator of lung metastasis in breast cancer and possibly other carcinomas, such as colon cancer, for which overexpression of Kindlin-1 was previously reported in a small cohort of 7 patients [16].

The Ki-67 index value is reportedly higher in malignant melanomas than in benign nevi, and this index has been correlated with prognosis in patients with melanoma. Conversely, other studies have not found a correlation between Ki-67 expression and overall survival $[5,21,22]$.

There is significant heterogeneity between studies with regard to immunohistochemistry techniques, analysis methods and number of tissue samples tested. Although our study did not reveal correlations between the markers tested and risk factors or clinicopathological parameters, some previous results have shown correlations between Ki-67 and prognostic factors in cutaneous melanoma. We used a continuous variable (percentage) to measure Ki-67 staining in tissue samples, as there is no standard method in the literature for assessing Ki-67 positivity in primary melanoma.

We did not find any previous studies involving Kindlin-1 staining in primary melanomas, but there are some studies correlating Kindlin-3 and melanomas or Kindlin-1 staining and prognosis for other cancers, including skin cancer. Overall, our analysis did not show any correlations between Kindlin-1 and the clinicopathological parameters we examined.

\section{Conclusions}

In our study, we did not compare primary melanoma samples and metastatic disease or dysplastic nevi, as previously conducted $[10,14,15]$. We believe that differences in marker expression may occur in each condition. Beyond this limitation, a larger cohort may find different results. We believe that additional studies are also necessary to assess whether expression of these markers correlates with clinical outcomes in patients with cutaneous melanoma.

\section{Abbreviation}

AJCC: American Joint Committee on Cancer; BSA: Bovin serum albumin; FERMT1: Fermitin family member 1; IHC: Imunohistochemistry;

ISCMPA: Irmandade Santa Casa de Misericórdia de Porto Alegre;

PBS: Phosphate buffered saline; UFCSPA: Universidade Federal de Ciências da Saúde de Porto Alegre; USA: United States of America

Acknowledgements

To the pathology laboratory of UFCSPA. 


\section{Authors' contributions}

LEHS, MAM and CGB developed the project, selected and analyzed patients data and contributed to statistical analysis. RF and PR performed and interpreted all histological examination. KCR and FR interviewed patients and performed immunohistochemistry and technical analysis. IC was a major contributor to statistical analysis and organized tables and figures. All authors read and approved the final manuscript.

\section{Funding}

Not applicable.

\section{Availability of data and materials}

Data of thirty patients from the files of patients diagnosed and treated in our institution (ISCMPA).

\section{Ethics approval and consent to participate}

Written informed consent was obtained from each patient according to the recommendations of the local ethics committee. This study was approved by the ethics committee of the hospital and the university where the study was developed (ISCMPA and UFCSPA, respectively).

\section{Consent for publication}

All authors are aware of this publication.

\section{Competing interests}

The authors declare that they have no competing interests.

\section{Author details}

${ }^{1}$ Universidade Federal de Ciências da Saúde de Porto Alegre, Porto Alegre, Brazil. ${ }^{2}$ Hospital Santa Rita, Irmandade Santa Casa de Misericórdia de Porto Alegre, Porto Alegre, Brazil. ${ }^{3}$ Pontifícia Universidade Católica do Rio Grande do Sul, Porto Alegre, Brazil.

Received: 21 May 2019 Accepted: 28 July 2019

Published online: 02 September 2019

\section{References}

1. American Cancer Society. Cancer facts and figures. Atlanta: ACS; 2016. Available at www.cancer.org. Accessed 28 Nov 2016

2. Grazziotin TC, Rey MC, Bica CG, et al. Genetic variations of patients with familial or multiple melanoma in southern Brazil. J Eur Acad Dermatol Venereol. 2013;27:179-85.

3. Instituto Nacional do Câncer. Tipos de Câncer. Rio de Janeiro: INCA; 2016. Available at www.inca.gov.br. Accessed 28 Nov 2016

4. Pieniazek M, Donizy P, Halon A, Leskiewicz M, Matkowski R. Prognostic significance of immunohistochemical epitelial-mesenchymal transition markers in skin melanoma patients. Biomark Med. 2016;10(9):975-85.

5. Uguen A, Talagas M, Costa S, et al. A p16-Ki- 67-HMB45 immunohistochemistry scoring system as an ancillary diagnostic tool in the diagnosis of melanoma. Diagn Pathol. 2015;10:195-9.

6. Reid AL, Millward M, Pearce R, et al. Markers of circulating tumour cells in the peripheral blood of patients with melanoma correlate with disease recurrence and progression. Br J Dermatol. 2013;168(1):85-92.

7. Balch CM, Gershenwald JE, Soong S, et al. Final version of 2009 AJCC melanoma staging and classification. J Clin Oncol. 2009;27(36):6199-206.

8. Li N, Mangini J, Li N, Mangini J, Bhawan J. New prognostic factors of cutaneous jag Bhawan melanoma: a review of the literature. J Cutan Pathol. 2002;29:324-40

9. Gimotty PA, Botbyl J, Soong SJ, Guerry D. A population based validation of the American joint committee on Cancer melanoma staging system. J Clin Oncol. 2005;23:8065-75.

10. Chen T, Zhu J. Evaluation of EMMPRIN and MMP-2 in the prognosis of primary cutaneous malignant melanoma. Med Oncol. 2010;27:1185-91.

11. Chen G, Cheng Y, Zhang Z, Martinka M, Li G. Prognostic significance of cytoplasmic p27 expression in human melanoma. Cancer Epidemiol Biomark Prev. 2011;20:2212-21.

12. Vereecken $P$, Laporte M, Heenen M. Significance of cell kinetic parameters in the prognosis of malignant melanoma: a review. J Cutan Pathol. 2007;34: $139-45$.
13. Henrique R, Azevedo R, Bento MJ, Domingues JC, Silva C, Jeronimo C. Prognostic value of Ki-67 expression in localized cutaneous malignant melanoma. J Am Acad Dermatol. 2000;43:991-1000.

14. Gould Rothberg BE, Bracken MB, Rimm DL. Tissue biomarkers for prognosis in cutaneous melanoma: a systematic review and meta-analysis. J Natl Cancer Inst. 2009;101:452-74.

15. Cheng Y, Lu J, Chen G, et al. Stage-specific prognostic biomarkers in melanoma. Oncotarget. 2015;6:4180-9.

16. Ohsie SJ, Sarantopoulos GP, Cochran AJ, Binder SW. Immunohistochemical characteristics of melanoma. J Cutan Pathol. 2008;35:433-44.

17. Landemaine $\mathrm{T}$, Jackson $\mathrm{A}$, Bellahcene $\mathrm{A}$, et al. A six-gene signature predicting breast cancer lung metastasis. Cancer Res. 2008;68:6092-6099.10.

18. Plow EF, Das M, Bialkowska K, Sossey-Alaoui K. Of Kindlins and Cancer. Discoveries. 2016;4(2):59.

19. Djaafri I, Khayati F, Menashi S, et al. A novel tumor suppressor function of kindlin-3 in solid cancer. Oncotarget. 2014;19(5):8970-85.

20. Sin S, Bonin F, Petit V, et al. Role of the focal adhesion protein kindlin-1 in breast cancer growth and lung metastasis. J Natl Cancer Inst. 2011;103: 1323-37.

21. Weinstein EJ, Bourner M, Head R, Zakeri H, Bauer C, Mazzarella R. URP1: a member of a novel family of PH and FERM domain-containing membraneassociated proteins is significantly over-expressed in lung and colon carcinomas. Biochim Biophys Acta. 2003;1637(3):207-16.

22. Mahawithitwong $P$, Ohuchida K, Ikenaga N, et al. Kindlin-1 expression is involved in migration and invasion of pancreatic cancer. Int J Oncol. 2013; 42:1360-6.

23. Gerdes J, Schwab U, Lemke H, Stein H. Production of a mouse monoclonal antibody reactive with a human nuclear antigen associated with cell proliferation. Int J Cancer. 1983;31(1):13-20.

24. Prieto VG, Shea CR. Use of immunohistochemistry in melanocytic lesions. J Cutan Pathol. 2008:35:1-10.

25. Wandler A, Spaun E, Steiniche T. Automated quantification of Ki67/MART1 stains may prevent false-negative melanoma diagnoses. J Cutan Pathol. 2016;43:956-62.

26. Nielsen PS, Riber-Hansen R, Jensen TO, Schmidt H, Steiniche T. Proliferation indices of phosphohistone $\mathrm{H} 3$ and Ki67: strong prognostic markers in a consecutive cohort with stage 1/II melanoma. Mod Pathol. 2013:26:404-13.

27. Swiderska Z, Korzynska A, Markiewicz T, Lorent M, Zak J, Wesolowska A, et al. Comparison of the manual, semiautomatic, and automatic selection and leveling of hot spots in whole slide images for Ki-67 quantification in meningiomas. Anal Cell Pathol (Amst). 2015;2015:498746.

28. Mei-Yin C, Polley Samuel CY, Leung Lisa M, McShane Dongxia Gao Judith C, Hugh Mauro G, Mastropasqua Giuseppe Viale Lila A, Zabaglo Frédérique Penault-Llorca John MS, Bartlett. An International Ki67 Reproducibility Study. J Natl Cancer Inst. 2013;105(24):1897-1906. https://doi.org/10.1093/jnci/djt306.

\section{Publisher's Note}

Springer Nature remains neutral with regard to jurisdictional claims in published maps and institutional affiliations.

Ready to submit your research? Choose BMC and benefit from:

- fast, convenient online submission

- thorough peer review by experienced researchers in your field

- rapid publication on acceptance

- support for research data, including large and complex data types

- gold Open Access which fosters wider collaboration and increased citations

- maximum visibility for your research: over $100 \mathrm{M}$ website views per year

At $\mathrm{BMC}$, research is always in progress.

Learn more biomedcentral.com/submissions 